\title{
STABILITY AND WELL-POSEDNESS IN LINEAR SEMI-INFINITE PROGRAMMING*
}

\author{
M. J. CÁNOVAS ${ }^{\dagger}$, M. A. LÓPEZ ${ }^{\ddagger}$, J. PARRA ${ }^{\dagger}$, AND M. I. TODOROV $\S$
}

\begin{abstract}
This paper presents an approach to the stability and the Hadamard well-posedness of the linear semi-infinite programming problem (LSIP). No standard hypothesis is required in relation to the set indexing of the constraints and, consequently, the functional dependence between the linear constraints and their associated indices has no special property. We consider, as parameter space, the set of all LSIP problems whose constraint systems have the same index set, and we define in it an extended metric to measure the size of the perturbations. Throughout the paper the behavior of the optimal value function and of the optimal set mapping are analyzed. Moreover, a certain type of Hadamard well-posedness, which does not require the boundedness of the optimal set, is characterized. The main results provided in the paper allow us to point out that the lower semicontinuity of the feasible set mapping entails high stability of the whole problem, mainly when this property occurs simultaneously with the boundedness of the optimal set. In this case all the stability properties hold, with the only exception being the lower semicontinuity of the optimal set mapping.
\end{abstract}

Key words. stability, Hadamard well-posedness, semi-infinite programming, feasible set mapping, optimal set mapping, optimal value function

AMS subject classifications. 15A39, 49D39, 49M39, 52A40, 65F99, 90C34

PII. S1052623497319869

1. Introduction. We consider the linear optimization problem in $\mathbb{R}^{n}$ :

$$
\pi: \quad \operatorname{Inf}\left\{c^{\prime} x \mid a_{t}^{\prime} x \geq b_{t}, t \in T\right\}
$$

where $c, x$, and $a_{t}$ belong to $\mathbb{R}^{n}, b_{t} \in \mathbb{R}$, and $y^{\prime}$ denotes the transpose of $y \in \mathbb{R}^{n} . \pi$ is alternatively represented by the couple $(c, \sigma)$, or by $\left(c,\left(a_{t}, b_{t}\right)_{t \in T}\right)$.

If the index set $T$ of the constraints system, $\sigma:=\left\{a_{t}^{\prime} x \geq b_{t}, t \in T\right\}$, is infinite, we have a linear semi-infinite programming problem (LSIP). We shall not assume any structure for $T$ and, consequently, the functions $t \mapsto a_{t}$ and $t \mapsto b_{t}$ have no particular property.

The parameter space, in our approach, is the set $\Pi$ of all the problems $\pi=$ $(c, \sigma)$, with $c \neq 0_{n}$, whose constraint systems have the same index set $T$. When different problems are considered in $\Pi$, they and their associated elements will be distinguished by means of sub- and superscripts. So, if $\pi_{1}$ also belongs to $\Pi$, we write $\pi_{1}=\left(c^{1}, \sigma_{1}\right)$ and $\sigma_{1}:=\left\{\left(a_{t}^{1}\right)^{\prime} x \geq b_{t}^{1}, t \in T\right\}$. Obviously, we can identify $\Pi$ with $\left(\mathbb{R}^{n} \backslash\left\{0_{n}\right\}\right) \times\left(\mathbb{R}^{n} \times \mathbb{R}\right)^{T}$, where the set of possible systems is itself identified with $\left(\mathbb{R}^{n} \times \mathbb{R}\right)^{T}$.

*Received by the editors April 11, 1997; accepted for publication (in revised form) September 10, 1998; published electronically October 20, 1999. This research was partially supported by grants PB95-0687 and SAB 95-0311 from DGES and by grants GV-2219/94 and GV-C-CN-10-067-96 from Generalitat Valenciana.

http://www.siam.org/journals/siopt/10-1/31986.html

${ }^{\dagger}$ Department of Statistics and Applied Mathematics, Miguel Hernández University, 03202 Elche (Alicante), Spain (canovas@umh.es, parra@umh.es).

${ }^{\ddagger}$ Department of Statistics and Operations Research, University of Alicante, 03071 Alicante, Spain (marco.antonio@ua.es).

$\S$ Bulgarian Academy of Sciences, Institute of Mathematics, 29 Ph. Macedonsky Str., r. 401, 4002 Plovdiv, Bulgaria (todorovm@bgearn.bitnet). 
Many LSIP problems have coefficients whose values either are known only approximately or have to be rounded off in the computing process. Therefore, we actually solve a different problem, $\pi_{1}=\left(c^{1}, \sigma_{1}\right)$, proximal to the original one, $\pi=(c, \sigma)$. An extended distance $\delta: \Pi \times \Pi \rightarrow[0,+\infty]$ is introduced by means of

$$
\delta\left(\pi_{1}, \pi\right):=\max \left\{\left\|c^{1}-c\right\|_{\infty}, \sup _{t \in T}\left\|\left(\begin{array}{c}
a_{t}^{1} \\
b_{t}^{1}
\end{array}\right)-\left(\begin{array}{c}
a_{t} \\
b_{t}
\end{array}\right)\right\|_{\infty}\right\} .
$$

$(\Pi, \delta)$ is a Hausdorff space, whose topology satisfies the first axiom of countability (i.e., convergence is established by means of sequences, since each point has a countable base of neighborhoods), and describes the uniform convergence topology on $\Pi$. If $T$ is a compact Hausdorff space and the functions $t \mapsto a_{t}$ and $t \mapsto b_{t}$ are continuous, $\pi$ is said to be continuous. We shall denote by $\Pi_{o}$ the set of continuous LSIP problems. $\left(\left(\Pi_{o}, \delta\right)\right.$ is a metric space. $)$

In this paper, we study the stability properties of $\pi$. More precisely, we analyze the lower and upper semicontinuity of the optimal value function, $\vartheta$, and the optimal set mapping, $\mathcal{F}^{*}$. The former assigns to each problem $\pi$ its optimal value $v$ (i.e., $\vartheta(\pi)=v$ ), and the latter assigns to $\pi$ the (possibly empty) optimal set, represented by $F^{*}$ (i.e., $\mathcal{F}^{*}(\pi)=F^{*}$ ). We prove that the lower semicontinuity of the feasible set mapping, $\mathcal{F}$, assigning to $\pi$ the (possibly empty) feasible set $F$ (i.e., $\mathcal{F}(\pi)=F$ ), and the boundedness of $F^{*}$ (especially when both hold simultaneously), yield nice stability properties of $\vartheta$ and $\mathcal{F}^{*}$ at $\pi$. So, we devote section 3 to presenting different characterizations of the lower semicontinuity of $\mathcal{F}$ at $\pi$, which are used throughout the paper. As a counterpart of the important lower semicontinuity property, Lemma 4.1 states that the boundedness of the optimal set, assumed to be nonempty, is equivalent to a certain stability of $\pi$ : any sufficiently close problem, with nonempty feasible set, also has optimal solutions.

Section 4 contains the main results concerning the optimal value function. Theorem 4.2 deals with the continuity properties of $\vartheta$, whereas in the second part of this section we propose a definition of Hadamard well-posedness, based on the strategy of solving, in an approximated way, the sequence of problems approaching $\pi$. Our concept of Hadamard well-posedness, which does not require the uniqueness of the optimal solution, is oriented toward the stability of the optimal value function and can be traced out from Dontchev and Zolezzi [4]. Theorem 4.3 delimits the scope of this new concept.

Section 5 focuses on the stability behavior of the optimal set mapping, $\mathcal{F}^{*}$. Theorem 5.1 clarifies the role played by the closedness of this mapping. At the end of section 5, Table 5.1 summarizes the theory developed in the paper, emphasizing the importance of the lower semicontinuity of $\mathcal{F}$ at $\pi$, and of the boundedness of $F^{*}$, in the global stability of $\pi$. Section 6 supplies examples showing that every unfixed possibility in Table 5.1 can actually occur.

Some statements in sections 4 and 5 constitute extensions to the general LSIP of different results obtained by Brosowski [2] and Fischer [5] for the continuous LSIP. Moreover, in a forthcoming paper [3], we prove that, under the unicity of the optimal solution, our concept of Hadamard well-posedness is equivalent to other concepts [17] that, at first glance, seem much more restrictive.

2. Preliminaries. The optimal value function $\vartheta$ will take values in $[-\infty,+\infty]$ if we define $\vartheta(\pi)=+\infty$ when $\pi$ is inconsistent (i.e., when $F=\emptyset$ ) and $\vartheta(\pi)=-\infty$ when $\pi$ is unbounded (i.e., when $c^{\prime} x$ is not bounded from below on $F$ ). Hereafter, $\Pi_{c}$ represents the consistent problems subset $\left(\pi \in \Pi_{c} \Leftrightarrow F \neq \emptyset \Leftrightarrow \vartheta(\pi)<+\infty\right)$, and $\Pi_{b}$ 
denotes the set of bounded problems $\left(\pi \in \Pi_{b} \Leftrightarrow \vartheta(\pi)\right.$ is finite). In addition, $\Pi_{s}$ will be the set of solvable problems $\left(\pi \in \Pi_{s} \Leftrightarrow F^{*} \neq \emptyset \Leftrightarrow \vartheta(\pi)\right.$ is attained). Obviously, $\Pi_{s} \subset \Pi_{b} \subset \Pi_{c}$.

At this point we introduce some necessary notation. Given $\emptyset \neq X \subset \mathbb{R}^{p}$, by $\operatorname{conv}(X)$, cone $(X), O^{+}(X)$, and $X^{o}$ we denote the convex hull of $X$, the conical convex hull of $X$, the recession cone of $X$ (assuming that $X$ is convex), and the dual cone of $X$ (i.e., $X^{o}=\left\{y \in \mathbb{R}^{p} \mid y^{\prime} x \geq 0\right.$ for all $\left.x \in X\right\}$ ), respectively. It is assumed that cone $(X)$ always contains the zero-vector, and so cone $(\emptyset)=\left\{0_{n}\right\}$. The Euclidean and Chebyshev norms of $x \in \mathbb{R}^{p}$ will be $\|x\|$ and $\|x\|_{\infty}$, respectively, and the Euclidean distance from $x$ to $X(\neq \emptyset)$ is $d(x, X):=\inf \{\|x-y\|: y \in X\}$. The unit open ball, in $\mathbb{R}^{p}$, for the Euclidean norm is represented by $B$. From the topological side, if $X$ is a subset of any topological space, $\operatorname{int}(X), \operatorname{cl}(X)$, and $\mathrm{bd}(X)$ represent the interior, the closure, and the boundary of $X$, respectively. Finally, $\lim _{r}$ should be interpreted as $\lim _{r \rightarrow \infty}$.

If $\left\{X_{r}\right\}$ is a sequence of nonempty sets in $\mathbb{R}^{p}, \liminf _{r} X_{r}\left(\limsup _{r} X_{r}\right)$ is the set of all the limits (cluster points) of all the possible sequences $\left\{x^{r}\right\}, x^{r} \in X_{r}, r=1,2, \ldots$, and it can be characterized as the set of points $x$ such that every neighborhood of $x$ intersects all the sets $X_{r}$ except a finite number of them (it intersects infinitely many sets $X_{r}$ ). It is said that $\left\{X_{r}\right\}$ converges to $X$, in the Painlevé-Kuratowski sense (see, for instance, [15]) if $X=\liminf _{r} X_{r}=\lim \sup _{r} X_{r}$. In this case we write $X=\lim _{r} X_{r}$.

Next we recall some well-known continuity concepts for set-valued mappings. If $\mathcal{Y}$ and $\mathcal{Z}$ are two topological spaces and $\mathcal{S}: \mathcal{Y} \rightarrow 2^{\mathcal{Z}}$ is a set-valued mapping, we shall consider the following properties of $\mathcal{S}$.

If both spaces verify the first axiom of countability, we say that $\mathcal{S}$ is closed at $y \in \mathcal{Y}$ if for all sequences $\left\{y^{r}\right\} \subset \mathcal{Y}$ and $\left\{z^{r}\right\} \subset \mathcal{Z}$ satisfying $\lim _{r} y^{r}=y, \lim _{r} z^{r}=z$, and $z^{r} \in \mathcal{S}\left(y^{r}\right)$, one has $z \in \mathcal{S}(y)$.

The mapping $\mathcal{S}$ is lower semicontinuous (lsc) at $y \in \mathcal{Y}$ if for each open set $W \subset \mathcal{Z}$ such that $W \cap \mathcal{S}(y) \neq \emptyset$, there exists an open set $U \subset \mathcal{Y}$, containing $y$, such that $W \cap \mathcal{S}\left(y^{1}\right) \neq \emptyset$ for each $y^{1} \in U$.

$\mathcal{S}$ is said to be upper semicontinuous (usc) at $y \in \mathcal{Y}$ if for each open set $W \subset \mathcal{Z}$ such that $\mathcal{S}(y) \subset W$, there exists an open neighborhood of $y$ in $\mathcal{Y}, U$, such that $\mathcal{S}\left(y^{1}\right) \subset W$ for every $y^{1} \in U$.

Given a consistent system $\sigma:=\left\{a_{t}^{\prime} x \geq b_{t}, t \in T\right\}$, with solution set $F$, we say that $a^{\prime} x \geq b$ is a consequence of $\sigma$ if it is satisfied at each point of $F$, i.e., if $a^{\prime} z \geq b$ for every $z \in F$.

Throughout this paper we shall apply the so-called nonhomogeneous Farkas lemma [19], which characterizes the linear inequalities $a^{\prime} x \geq b$ that are consequences of a consistent system $\sigma:=\left\{a_{t}^{\prime} x \geq b_{t}, t \in T\right\}$ as those satisfying

$$
\left(\begin{array}{l}
a \\
b
\end{array}\right) \in \operatorname{cl}\left(\text { cone }\left(\left\{\left(\begin{array}{l}
a_{t} \\
b_{t}
\end{array}\right), t \in T ;\left(\begin{array}{c}
0_{n} \\
-1
\end{array}\right)\right\}\right)\right) \text {. }
$$

If we introduce the cone, $\mathbb{R}_{+}^{(T)}$, of all the functions $\lambda: T \rightarrow \mathbb{R}_{+}$taking positive values only at finitely many points of $T,(2.1)$ is equivalent to the existence of sequences $\left\{\lambda^{r}\right\} \subset \mathbb{R}_{+}^{(T)}$ and $\left\{\mu_{r}\right\} \subset \mathbb{R}_{+}$, such that

$$
\left(\begin{array}{l}
a \\
b
\end{array}\right)=\lim _{r}\left\{\sum_{t \in T} \lambda_{t}^{r}\left(\begin{array}{l}
a_{t} \\
b_{t}
\end{array}\right)+\mu_{r}\left(\begin{array}{c}
0_{n} \\
-1
\end{array}\right)\right\},
$$

where $\lambda^{r}=\left(\lambda_{t}^{r}\right)_{t \in T}, r=1,2, \ldots$ 
3. Feasible set mapping. In $[7$, sect. 2] it is proved that the mapping $\mathcal{F}$ is always closed at any $\pi \in \Pi_{c}$. In that paper, and also in [6], different characterizations of the lower semicontinuity of $\mathcal{F}$ at a consistent problem $\pi$ are provided, most of them based upon different stability concepts taken from the literature ([11], [14], [18], etc.). The following theorem gathers some of these characterizations and adds some new ones, which will be applied below. We recall here the strong Slater condition (SS condition), which is satisfied by $\pi$ if there exist a positive scalar $\rho$ and a feasible point $\bar{x}$ satisfying $a_{t}^{\prime} \bar{x} \geq b_{t}+\rho$ for all $t \in T(\bar{x}$ is called an SS element of $\sigma$ ). The SS condition is certainly stronger than the well-known Slater condition, which only requires the existence of a strict solution, $\bar{x}$, satisfying $a_{t}^{\prime} \bar{x}>b_{t}$ for all $t \in T$ (obviously, if $\pi$ is continuous, both conditions are equivalent). The set of all the SS elements of $\sigma$ will be represented by $F_{S S}$.

THEOREM 3.1. If $\pi=(c, \sigma) \in \Pi_{c}$, then the following statements are equivalent:

i. $\mathcal{F}$ is lsc at $\pi$;

ii. $\pi \in$ int $\left(\Pi_{c}\right)$;

iii. $0_{n+1} \notin \operatorname{cl}\left(\operatorname{conv}\left(\left\{\left(\begin{array}{c}a_{t} \\ b_{t}\end{array}\right), t \in T\right\}\right)\right)$;

iv. $\pi$ satisfies the $\mathrm{SS}$ condition;

v. For every sequence $\left\{\pi_{r}\right\} \subset \Pi$ converging to $\pi$, there exists an $r_{0}$ such that $\pi_{r} \in \Pi_{c}$ if $r \geq r_{0}$, and $F=\lim _{r \geq r_{0}} F_{r}$;

vi. $F=\operatorname{cl}\left(F_{S S}\right)$.

Proof. The equivalence between the first four statements is established in [7, Thm. 3.1]. Next we prove the equivalence of statements $i$ and $v$. Let us assume first that statement i holds. Since i $\Leftrightarrow$ ii has already been established, from $\pi \in \operatorname{int}\left(\Pi_{c}\right)$ we conclude the existence of $r_{0}$ such that $F_{r} \neq \emptyset$ if $r \geq r_{0}$. Then, if $x \in F$ and $W$ is an open neighborhood of $x$, statement i yields $r_{1}\left(r_{1} \geq r_{0}\right)$ such that $W \cap F_{r} \neq \emptyset$ for all $r \geq r_{1}$. In other words, $W$ intersects all the sets $F_{r}$, except a finite number of them, which identifies $x$ as a point of $\liminf _{r \geq r_{0}} F_{r}$. Moreover, $\lim \sup _{r \geq r_{0}} F_{r} \subset F$ because $\mathcal{F}$ is closed at every $\pi$. Since the inclusion $\liminf _{r \geq r_{0}} F_{r} \subset \limsup _{r \geq r_{0}} F_{r}$ always holds, one concludes that $F=\lim _{r \geq r_{0}} F_{r}$.

We proceed by assuming that part $\mathrm{v}$ holds and statement $\mathrm{i}$ fails. This implies the existence of an open set $W$ such that $F \cap W \neq \emptyset$, whereas for each $r \in \mathbb{N}$ we can find $\pi_{r}$ such that $\delta\left(\pi_{r}, \pi\right)<1 / r$ and $F_{r} \cap W=\emptyset$. Consequently, if $x \in F \cap W$, and whichever $r_{0}$ we consider, $x \notin \liminf _{r \geq r_{0}} F_{r}$. Thus, $\lim _{r} \pi_{r}=\pi$ and $F \neq \liminf _{r \geq r_{0}} F_{r}$ for every $r_{0}$, contradicting the assumption.

Next we prove i $\Leftrightarrow$ vi. If statement vi holds, since $F \neq \emptyset$ by hypothesis, $F_{S S}$ must be nonempty too, and we apply the equivalence between statements i and iv, already established. Conversely, if statement $\mathrm{i}$ is held, given any open set $W$ intersecting $F$, we can find $\bar{\rho}>0$ such that $F_{1} \cap W \neq \emptyset$ if $\pi_{1}:=\left(c, \sigma_{1}\right)$ with $\sigma_{1}:=\left\{a_{t}^{\prime} x \geq b_{t}+\bar{\rho}, t \in T\right\}$. Since $F_{1}=\mathcal{F}\left(\pi_{1}\right) \subset F_{S S}$, we obtain $F_{S S} \cap W \neq \emptyset$. We have just proved that $F \cap W \neq \emptyset$ implies $F_{S S} \cap W \neq \emptyset$, which itself implies $F \subset \operatorname{cl}\left(F_{S S}\right)$. The opposite inclusion comes from the trivial relation $F_{S S} \subset F$.

Concerning the upper semicontinuity of $\mathcal{F}$ at $\pi \in \Pi_{c}$, in the characterization given in [8, Thm. 3.1], the boundedness of $F$ (see [5]) is not required any longer, although this condition is still sufficient [8, Cor. 3.2]. If $n \geq 2$ and $\left\{a_{t}, t \in T\right\}$ is bounded and different from $\left\{0_{n}\right\}, \mathcal{F}$ will be usc at $\pi \in \Pi_{c}$ if and only if $F$ is bounded [8, Thm. 3.4]. Finally, in the case $n=1$, it is remarked in [8, Ex. 3.3] that $\mathcal{F}$ is always usc at every consistent problem. 
When we confine ourselves to continuous problems, we shall denote by $\Pi_{o c}$ the set of consistent continuous LSIP problems in $\mathbb{R}^{n}$, all of them having constraint systems indexed by a compact Hausdorff space $T$. It was proved in [7, Thm. 6.2] that the restriction of $\mathcal{F}$ to $\Pi_{o}$, represented by $\mathcal{F}_{o}$, is lsc at $\pi \in \Pi_{o c}$ if and only if $\sigma$ satisfies the well-known Slater condition or, equivalently, if $\pi$ belongs to $\operatorname{int}_{o}\left(\Pi_{o c}\right)$, the interior set of $\Pi_{o c}$ in the topology relative to $\Pi_{o}$. Moreover, and since $\left\{a_{t}, t \in T\right\}$ is compact when $\pi \in \Pi_{o}$, it turns out that, for $n \geq 2, \mathcal{F}_{o}$ is usc at $\pi \in \Pi_{o c}$ if and only if either $F$ is bounded or $F=\mathbb{R}^{n}$.

In section 4 we shall apply the following uniform metric regularity property.

LEMMA 3.2. Given $\pi \in \Pi_{c}$, assume that $\mathcal{F}$ is lsc at $\pi$ and that $F$ is bounded. Then, there exists a pair of positive scalars $\varepsilon$ and $\beta$ such that $\delta\left(\pi_{i}, \pi\right)<\varepsilon, i=1,2$, imply, for every $x^{j} \in F_{j}$,

$$
d\left(x^{j}, F_{i}\right) \leq \beta\left[\sup _{t \in T}\left\{b_{t}^{i}-\left(a_{t}^{i}\right)^{\prime} x^{j}\right\}\right]_{+}, \quad i, j=1,2, \quad i \neq j
$$

where $[\alpha]_{+}:=\max \{0, \alpha\}$.

Proof. The boundedness of $F$ implies that $\mathcal{F}$ is usc at $\pi$, and $\widehat{\varepsilon}>0$ exists such that $F_{1} \subset F+B$ if $\delta\left(\pi_{1}, \pi\right)<\widehat{\varepsilon}$. Thus we can find a positive scalar $\mu$ such that $\left\|x^{1}\right\| \leq \mu$ for every $x^{1} \in F_{1}$, provided that $\delta\left(\pi_{1}, \pi\right)<\widehat{\varepsilon}$. Moreover, it can be assumed, without loss of generality, that $F_{1} \neq \emptyset$ in this $\widehat{\varepsilon}$-neighborhood of $\pi$, because of the lower semicontinuity of $\mathcal{F}$ at $\pi$.

Now let us consider, in this neighborhood, two problems, $\pi_{1}$ and $\pi_{2}$. Take, for instance, an arbitrary $x^{2} \in F_{2}$ and suppose that $x^{2} \notin F_{1}$ (otherwise the inequality to be proved holds trivially). Suppose that $x^{1} \in F_{1}$ satisfies the $d\left(x^{2}, F_{1}\right)=\left\|x^{1}-x^{2}\right\|$.

Following a reasoning similar to that in [7, Thm. 3.1], we obtain

$$
d\left(x^{2}, F_{1}\right)=\left\|x^{1}-x^{2}\right\| \leq \frac{4 \mu}{\rho} \sup _{t \in T}\left\{b_{t}^{1}-\left(a_{t}^{1}\right)^{\prime} x^{2}\right\},
$$

provided that $\delta\left(\pi_{i}, \pi\right)<\varepsilon:=\min \left\{\widehat{\varepsilon}, \frac{\rho}{2}\left(1+n^{1 / 2} \mu\right)^{-1}\right\}, i=1,2$, where $\rho$ is the "slack" associated with an arbitrarily chosen SS element, $\bar{x}$, of $\sigma$ (i.e., $a_{t}^{\prime} \bar{x} \geq b_{t}+\rho$ for all $t \in T)$. We finish the proof by taking $\beta=\frac{4 \mu}{\rho}$.

There are, spread out in the literature, many contributions to the stability theory of $\mathcal{F}$ for a class of semi-infinite systems structurally richer than our linear inequality systems. This class is formed by those systems $\sigma$ whose index set $T$ is a compact set in the Euclidean space, defined as a solution set of finitely many analytic constraints. Moreover, the coefficient functions $a(\cdot)$ and $b(\cdot)$ are assumed to belong to $\mathcal{C}^{1}(T)$. Obviously, this class of $\mathcal{C}^{1}$-systems is a subclass of continuous systems.

Assuming that $\mathcal{C}^{1}(T)$ is equipped with the so-called Whitney topology, it is established in [11] that, under the assumption of the boundedness of $F$, $\mathcal{F}$ will be topologically stable at $\pi$ (homeomorphic feasible sets in a neigborhood of $\pi$ ) if and only if the Mangasarian-Fromovitz constraint qualification (MFCQ) is held. The extension of this result for an unbounded $F$ can be found in [10]. In this semi-infinite programming context (with $\mathcal{C}^{1}$ data), the equivalence between the MFCQ and the metric regularity of the constraints has been established in [9]. Parametric versions of these results are given in [12] and [13], again in the $\mathcal{C}^{1}$-data context (see also [16]). When one is confined to the context of linear data without any structure for $T$, the corresponding counterparts of these results were provided in [6] and [7], using ad hoc techniques based exclusively upon the semi-infinite version of the alternative theorems. (The analytic approach does not make sense in our context since nothing is known about the functions $a(\cdot)$ and $b(\cdot)$.) 
4. Optimal value function and Hadamard well-posedness. Let us consider the sublevel sets of the problem $\pi$ :

$$
L(\alpha):=\left\{x \in F \mid c^{\prime} x \leq \alpha\right\}=\left\{x \in \mathbb{R}^{n} \mid a_{t}^{\prime} x \geq b_{t}, t \in T ; c^{\prime} x \leq \alpha\right\}, \alpha \in \mathbb{R} .
$$

$L(\alpha)$ depends on $\pi$. So, the sublevel sets of a different problem $\pi_{1}$ will be denoted $L_{1}(\alpha)$.

Obviously, $O^{+}(L(\alpha))=\left\{y \in \mathbb{R}^{n} \mid a_{t}^{\prime} y \geq 0, t \in T ; c^{\prime} y \leq 0\right\}=\left\{a_{t}, t \in T ;-c\right\}^{o}$, which is independent of $\alpha$, so that all the nonempty sublevel sets will have the same recession cone.

In the following key lemma, $\operatorname{int}_{c}\left(\Pi_{s}\right)$ will represent the interior of $\Pi_{s}$ in the topology relative to $\Pi_{c}$. Theorem 2.7 in [5] can be obtained as an immediate corollary of this lemma together with Theorem 3.1.

Lemma 4.1. $\pi \in \operatorname{int}_{c}\left(\Pi_{s}\right)$ if and only if $F^{*}$ is a nonempty bounded set.

Proof. If $F^{*}$ is a nonempty bounded set, $O^{+}\left(F^{*}\right)=\left\{0_{n}\right\}=\left\{a_{t}, t \in T ;-c\right\}^{o}$ and, consequently, $0_{n} \in \operatorname{int}\left(\mathbb{R}^{n}\right)=\operatorname{int}\left(\right.$ cone $\left.\left(\left\{a_{t}, t \in T ;-c\right\}\right)\right)$. Now, let us note that if $\delta\left(\pi_{1}, \pi\right)$ is small enough one still has $0_{n} \in \operatorname{int}\left(\right.$ cone $\left.\left(\left\{a_{t}^{1}, t \in T ;-c^{1}\right\}\right)\right)[7$, Lem. 4.2]. Thus, by reversing our previous argument, we observe that every nonempty sublevel set of any consistent problem $\pi_{1}$ in a certain neighborhood of $\pi$ is bounded and, then, $F_{1}^{*}$ is nonempty $\left(c^{\prime} x\right.$ attains its minimum in a compact set).

Conversely, if $\pi=(c, \sigma) \in \operatorname{int}_{c}\left(\Pi_{s}\right)$ and $F^{*}$ is unbounded, we shall take $u \in$ $O^{+}\left(F^{*}\right), u \neq 0_{n}$, and then we shall construct the sequence of problems $\left\{\pi_{r}:=\right.$ $\left.\left(c-\frac{1}{r} u, \sigma\right)\right\}$.

Obviously, $\lim _{r} \pi_{r}=\pi$ and $\left\{\pi_{r}\right\} \subset \Pi_{c} \backslash \Pi_{b}$, because, whichever $x^{*} \in F^{*}$ we take, one has $x^{*}+\lambda u \in F^{*} \subset F=F_{r}$ for all $\lambda>0$, and $\lim _{\lambda \rightarrow \infty}\left(c-\frac{1}{r} u\right)^{\prime}\left(x^{*}+\lambda u\right)=$ $v-\frac{1}{r} u^{\prime} x^{*}-\lim _{\lambda \rightarrow \infty} \frac{\lambda}{r}\|u\|^{2}=-\infty$. Hence, the existence of such a sequence $\left\{\pi_{r}\right\}$ contradicts our current hypothesis.

The continuity properties of the optimal value function $\vartheta$ are established in the following theorem.

Theorem 4.2. Let $\pi=(c, \sigma) \in \Pi_{c}$. Then

i. $\mathcal{F}$ is lsc at $\pi$ if and only if $\vartheta$ is usc at $\pi$.

ii. If $F^{*}$ is a nonempty bounded set, $\vartheta$ will be lsc at $\pi$. If $\pi \in \Pi_{b}$, the converse statement holds.

iii. If $\mathcal{F}$ is lsc at $\pi$ and $F^{*}$ is a nonempty bounded set, then we can find positive scalars, $\eta$ and $k$, such that $\delta\left(\pi_{i}, \pi\right)<\eta, i=1,2$, yield the Lipschitzian inequality

$$
\left|\vartheta\left(\pi_{1}\right)-\vartheta\left(\pi_{2}\right)\right| \leq k \delta\left(\pi_{1}, \pi_{2}\right) .
$$

Proof. i. The "only if" part is a straightforward consequence of [4, Prop. 2, Chap. IX]. In order to prove the converse statement, let us consider that $\vartheta$ is usc at $\pi$. Let $\mu>v$. Then, there will exist $\eta>0$ such that $\delta\left(\pi_{1}, \pi\right)<\eta$ implies $v_{1} \leq \mu$ and, necessarily, $\pi_{1} \in \Pi_{c}$; i.e., $\pi \in \operatorname{int}\left(\Pi_{c}\right)$ and, so, $\mathcal{F}$ is lsc at $\pi$.

ii. Given the scalar $\varepsilon>0$, we have to prove that $\eta>0$ exists such that $\delta\left(\pi_{1}, \pi\right)<$ $\eta$ implies $v_{1} \geq v-\varepsilon$. If $\rho>0$ satisfies $F^{*} \subset \rho B$, we shall take the open set $W:=$ $\left\{x \in \mathbb{R}^{n} \mid c^{\prime} x>v-(\varepsilon / 2)\right\} \cap \rho B$. Obviously, $W \supset F^{*}$.

Let us consider the system

$$
\widetilde{\sigma}:=\left\{a_{t}^{\prime} x \geq b_{t}, t \in T ; c^{\prime} x \leq v\right\}
$$

with index set $\widetilde{T}:=T \cup\left\{t_{0}\right\}$, where $t_{0}$ is the index associated with the last inequality of $\widetilde{\sigma}\left(t_{0} \notin T\right)$. Obviously, its solution set, denoted by $\widetilde{F}$, coincides with $F^{*}$, which 
is a nonempty bounded set by assumption. Consequently, if we represent by $\widetilde{\mathcal{F}}$ the feasible set mapping defined on the parameter space $\widetilde{\Pi}$ of all the LSIP problems with constraint systems having $\widetilde{T}$ as index set, it follows that $\widetilde{\mathcal{F}}$ is usc at $\widetilde{\pi}:=(c, \widetilde{\sigma})$. Hence, $\widehat{\eta}>0$ exists such that, if $\widetilde{\delta}$ denotes the corresponding extended distance in $\widetilde{\Pi}$, $\widetilde{\delta}\left(\widetilde{\pi}_{1}, \widetilde{\pi}\right)<\widehat{\eta}$ will imply $\widetilde{F}_{1} \subset W$ (although $\widetilde{F}_{1}$ might be empty).

Let us take any problem $\pi_{1}=\left(c^{1}, \sigma_{1}\right)$ satisfying $\delta\left(\pi_{1}, \pi\right)<\eta$, with $\eta:=\min \{\widehat{\eta}$, $\left.\varepsilon /\left(2 \rho n^{1 / 2}\right)\right\}$. Define the associated problem in $\widetilde{\Pi}, \widetilde{\pi}_{1}=\left(c^{1}, \widetilde{\sigma}_{1}\right)$, where

$$
\widetilde{\sigma}_{1}:=\left\{\left(a_{t}^{1}\right)^{\prime} x \geq b_{t}^{1}, t \in T ;\left(c^{1}\right)^{\prime} x \leq v\right\} .
$$

(The right-hand side term of the last constraint is fixed at $v=\vartheta(\pi)$.) It is obvious that $\widetilde{\delta}\left(\widetilde{\pi}_{1}, \widetilde{\pi}\right)=\delta\left(\pi_{1}, \pi\right)<\eta \leq \widehat{\eta}$ and, so, $\widetilde{F}_{1}=L_{1}(v) \subset W$.

Two possibilities can arise. If $\widetilde{F}_{1}=\emptyset$, we have $v_{1} \geq v>v-\varepsilon$ (possibly, $v_{1}=+\infty$ ). Otherwise (i.e., when $\widetilde{F}_{1} \neq \emptyset$ ), if we take an arbitrary $x^{*} \in F_{1}^{*} \subset \widetilde{F}_{1}$, it can be written

$$
v_{1}=\left(c^{1}\right)^{\prime} x^{*}=c^{\prime} x^{*}+\left(c^{1}-c\right)^{\prime} x^{*}>v-\frac{\varepsilon}{2}-\left\|c^{1}-c\right\|_{\infty}\left\|x^{*}\right\| n^{1 / 2}>v-\varepsilon .
$$

Assume now that $\vartheta$ is lsc at $\pi \in \Pi_{b}$, and let us show that the level set $L(\mu)$, with $\mu>v$, is bounded, in which case $\pi$ will be solvable and $F^{*}$ bounded. Otherwise, we can take $u \in O^{+}(L(\mu)), u \neq 0_{n}$, and then construct the sequence $\left\{\pi_{r}:=\left(c-\frac{1}{r} u, \sigma\right)\right\}$. Obviously, $\lim _{r} \pi_{r}=\pi$ and, reasoning as in Lemma 4.1, we prove that $\left\{\pi_{r}\right\} \subset \Pi_{c} \backslash \Pi_{b}$, which contradicts our current hypothesis.

iii. By Theorem 3.1 and Lemma 4.1 there will exist $\widehat{\eta}>0$ such that $\delta\left(\pi_{1}, \pi\right)<\widehat{\eta}$ implies $\pi_{1} \in \Pi_{s}$. Given $\varepsilon>0$, the upper semicontinuity of $\vartheta$ at $\pi$ guarantees that, if $\widehat{\eta}$ is small enough, one also has $v_{1} \leq v+\varepsilon$, which is equivalent in this case to $L_{1}(v+\varepsilon) \neq \emptyset$, provided that $\delta\left(\pi_{1}, \pi\right)<\widehat{\eta}$.

If we consider, instead of the system introduced in (4.1), the system

$$
\widetilde{\sigma}:=\left\{a_{t}^{\prime} x \geq b_{t}, t \in T ; c^{\prime} x \leq v+\varepsilon\right\},
$$

we observe that $\widetilde{F}=L(v+\varepsilon)$ is bounded (all the nonempty sublevel sets are bounded because $F^{*}=L(v)$ enjoys this property) and $\widetilde{\mathcal{F}}$ will again be usc at $\widetilde{\pi}$. Taking $\widehat{\eta}$ sufficiently small and $\pi_{1}=\left(c^{1}, \sigma_{1}\right)$ satisfying $\delta\left(\pi_{1}, \pi\right)<\widehat{\eta}$, we have

$$
L_{1}(v+\varepsilon) \subset L(v+\varepsilon)+B,
$$

since $L_{1}(v+\varepsilon)$ is the feasible set of $\widetilde{\pi}_{1}=\left(c^{1}, \widetilde{\sigma}_{1}\right)$, where

$$
\widetilde{\sigma}_{1}:=\left\{\left(a_{t}^{1}\right)^{\prime} x \geq b_{t}^{1}, t \in T ;\left(c^{1}\right)^{\prime} x \leq v+\varepsilon\right\}
$$

(note that $\left.\widetilde{\delta}\left(\widetilde{\pi}_{1}, \widetilde{\pi}\right)=\delta\left(\pi_{1}, \pi\right)\right)$.

Statement (4.2) means that $\mu>0$ can be found such that $\|x\| \leq \mu$ for all $x \in$ $L_{1}(v+\varepsilon)$ and for every $\pi_{1}$ in the $\widehat{\eta}$-neighborhood of $\pi$.

Applying Lemma 3.2 to $\widetilde{\pi}=(c, \widetilde{\sigma})$, we conclude the existence of $\eta>0$ (we shall take $\eta<\min \{1, \widehat{\eta}\})$ and $\beta>0$ such that, if $\pi_{i}=\left(c^{i}, \sigma_{i}\right), i=1,2$, are contained in the $\eta$-neighborhood of $\pi$ and, since $L_{i}(v+\varepsilon), i=1,2$, is the feasible set of $\widetilde{\pi}_{i}=\left(c^{i}, \widetilde{\sigma}_{i}\right)$, 


$$
\begin{gathered}
\widetilde{\sigma}_{i}:=\left\{\left(a_{t}^{i}\right)^{\prime} x \geq b_{t}^{i}, t \in T ;\left(c^{i}\right)^{\prime} x \leq v+\varepsilon\right\}, \text { one has for } x^{2} \in L_{2}(v+\varepsilon) \\
d\left(x^{2}, L_{1}(v+\varepsilon)\right) \leq \beta \max \left[\sup _{t \in T}\left\{b_{t}^{1}-\left(a_{t}^{1}\right)^{\prime} x^{2}\right\},\left(c^{1}\right)^{\prime} x^{2}-v-\varepsilon, 0\right] \\
=\beta \max \left[\sup _{t \in T}\left\{\left[b_{t}^{2}-\left(a_{t}^{2}\right)^{\prime} x^{2}\right]+\left[\left(b_{t}^{1}-b_{t}^{2}\right)-\left(a_{t}^{1}-a_{t}^{2}\right)^{\prime} x^{2}\right]\right\},\right. \\
\left.\left(c^{2}\right)^{\prime} x^{2}-v-\varepsilon+\left(c^{1}-c^{2}\right)^{\prime} x^{2}, 0\right] \\
\leq \beta \max \left[\sup _{t \in T}\left\{\left(b_{t}^{1}-b_{t}^{2}\right)-\left(a_{t}^{1}-a_{t}^{2}\right)^{\prime} x^{2}\right\},\left(c^{1}-c^{2}\right)^{\prime} x^{2}, 0\right] \\
\leq \beta\left(1+\mu n^{1 / 2}\right) \delta\left(\pi_{1}, \pi_{2}\right)=\beta_{0} \delta\left(\pi_{1}, \pi_{2}\right),
\end{gathered}
$$

where $\beta_{0}:=\beta\left(1+\mu n^{1 / 2}\right)$. Now, if $x^{2} \in F_{2}^{*} \subset L_{2}(v+\varepsilon)$, and taking $x^{1} \in L_{1}(v+\varepsilon)$ such that $\left\|x^{1}-x^{2}\right\|=d\left(x^{2}, L_{1}(v+\varepsilon)\right)$, it follows that

$$
\begin{aligned}
v_{1}-v_{2}= & v_{1}-\left(c^{2}\right)^{\prime} x^{2} \leq\left(c^{1}\right)^{\prime} x^{1}-\left(c^{2}\right)^{\prime} x^{2} \leq\left\|c^{1}-c^{2}\right\|\left\|x^{2}\right\|+\left\|c^{1}\right\|\left\|x^{1}-x^{2}\right\| \\
& \leq \mu n^{1 / 2} \delta\left(\pi_{1}, \pi_{2}\right)+n^{1 / 2}\left(\|c\|_{\infty}+\eta\right) \beta_{0} \delta\left(\pi_{1}, \pi_{2}\right) \leq k \delta\left(\pi_{1}, \pi_{2}\right),
\end{aligned}
$$

provided that $k:=n^{1 / 2}\left[\mu+\beta_{0}\left(\|c\|_{\infty}+1\right)\right]$.

Repeating the procedure for $v_{2}-v_{1}$, one obtains $\left|v_{1}-v_{2}\right| \leq k \delta\left(\pi_{1}, \pi_{2}\right)$.

In LSIP, existence and continuous dependence of the optimal solutions from problem's data might be established as follows.

Given $\left\{\pi_{r}=\left(c^{r}, \sigma_{r}\right)\right\} \subset \Pi_{b}$ such that $\lim _{r} \pi_{r}=\pi$, the sequence $\left\{x^{r}\right\}$ is said to be an asymptotically minimizing sequence (a.m.s.) for $\pi$ associated with $\left\{\pi_{r}\right\}$ if $x^{r} \in F_{r}$ for all $r$, and

$$
\lim _{r}\left\{\left(c^{r}\right)^{\prime} x^{r}-v_{r}\right\}=0
$$

i.e., as $r$ increases, $x^{r}$ approximately solves the approximating problem $\pi_{r}$.

The problem $\pi \in \Pi_{s}$ will be Hadamard well posed (Hwp) if for each $x^{*} \in F^{*}$ and for each possible sequence $\left\{\pi_{r}\right\} \subset \Pi_{b}$ converging to $\pi$, there exists at least an associated a.m.s. converging to $x^{*}$.

TheOREM 4.3. Given $\pi=(c, \sigma) \in \Pi_{s}$, the following statements hold:

i. If $\pi$ is Hwp, then the restriction of $\vartheta$ to $\Pi_{b}$, denoted by $\vartheta_{b}$, is continuous at $\pi$. If $\mathcal{F}$ is lsc at $\pi$, the converse statement is also true.

ii. Provided that $F^{*}$ is bounded, $\pi$ is Hwp if and only if either $\mathcal{F}$ is lsc at $\pi$ or $F$ is a singleton.

iii. When $F^{*}$ is unbounded and $\pi$ is Hwp, $\mathcal{F}$ has to be lsc at $\pi$.

Proof. i. First, we assume that $\pi$ is Hwp, and take $\left\{\pi_{r}\right\} \subset \Pi_{b}$ converging to $\pi$. We will see that $\lim _{r} v_{r}=v$.

The definition of Hadamard well-posedness states that, given $x^{*} \in F^{*}$, there will exist a sequence $\left\{x^{r}\right\}$ tending to $x^{*}$, such that $x^{r} \in F_{r}$ and $\lim _{r}\left\{\left(c^{r}\right)^{\prime} x^{r}-v_{r}\right\}=0$. Since $\lim _{r}\left(c^{r}\right)^{\prime} x^{r}=c^{\prime} x^{*}=v$, we obtain $\lim _{r} v_{r}=v$.

In order to prove the converse, we start from the continuity of $\vartheta_{b}$ at $\pi$ and from the lower semicontinuity of $\mathcal{F}$ at $\pi$. If $\left\{\pi_{r}\right\} \subset \Pi_{b}$ converges to $\pi$ and $x^{*} \in F^{*} \subset F$, the lower semicontinuity of $\mathcal{F}$ at $\pi$ implies $x^{*} \in \liminf _{r} F_{r}$ (condition $\mathrm{v}$ in Theorem 3.1). In other words, there must exist a sequence $\left\{x^{r}\right\}$ converging to $x^{*}$ and such that $x^{r} \in F_{r}$. Then $\left\{x^{r}\right\}$ turns out to be an a.m.s. for $\pi$ associated with $\left\{\pi_{r}\right\}$, since $\lim _{r}\left\{\left(c^{r}\right)^{\prime} x^{r}-v_{r}\right\}=0$.

ii. Assume that $\pi$ is Hwp, that $F^{*}$ is bounded, and that, at the same time, $F$ is not a singleton and $\mathcal{F}$ fails to be lsc at $\pi$. 
Pick an optimal point $x^{*} \in F^{*}$ and an arbitrary $y \in F \backslash\left\{x^{*}\right\}$. Define $u:=y-x^{*}$ and, associated with each $r \in \mathbb{N}$, take a positive scalar $k_{r}$ satisfying

$$
\left\|\frac{1}{k_{r}} u\right\|_{\infty}<\frac{1}{r} \quad \text { and } \quad\left|\frac{1}{k_{r}} u^{\prime} y\right|<\frac{1}{r} .
$$

According to condition iii in Theorem 3.1, the unfulfillment of the lower semicontinuity of $\mathcal{F}$ at $\pi$ gives rise to the existence of a sequence $\left\{\lambda^{p}\right\} \subset \mathbb{R}_{+}^{(T)}$, satisfying $\sum_{t \in T} \lambda_{t}^{p}=1, p=1,2, \ldots$, and

$$
0_{n+1}=\lim _{p} \sum_{t \in T} \lambda_{t}^{p}\left(\begin{array}{c}
a_{t} \\
b_{t}
\end{array}\right) .
$$

Let us introduce, for each $r \in \mathbb{N}$, the problem $\pi_{r}=\left(c, \sigma_{r}\right)$ with

$$
\sigma_{r}:=\left\{\left(a_{t}+\frac{1}{k_{r}} u\right)^{\prime} x \geq b_{t}+\frac{1}{k_{r}} u^{\prime} y, t \in T\right\} .
$$

Obviously, $\delta\left(\pi_{r}, \pi\right)<\frac{1}{r}$ and, so, $\lim _{r} \pi_{r}=\pi$. Moreover, $y \in F_{r}$ for every $r$, and $u^{\prime} x \geq u^{\prime} y$ is a consequence of each $\sigma_{r}$, since (4.3) implies

$$
\lim _{p} \sum_{t \in T} \lambda_{t}^{p}\left(\begin{array}{c}
a_{t}+\frac{1}{k_{r}} u \\
b_{t}+\frac{1}{k_{r}} u^{\prime} y
\end{array}\right)=\frac{1}{k_{r}}\left(\begin{array}{c}
u \\
u^{\prime} y
\end{array}\right)
$$

According to Lemma 4.1, the boundedness of $F^{*}$ entails that $\left\{\pi_{r}\right\}_{r \geq m} \subset \Pi_{s}$ for a certain $m$.

We have realized that $u^{\prime} x \geq u^{\prime} y$ for every $x \in F_{r}$, but $u^{\prime}\left(x^{*}-y\right)=-\|u\|^{2}$ and, so, $u^{\prime} x^{*}<u^{\prime} y$. This implies that, for this optimal point $x^{*}$ and for this particular sequence of bounded problems converging to $\pi$, there is no associated a.m.s. $\left\{x^{r}\right\}_{r \geq m}$ converging to $x^{*}$, and the Hadamard well-posedness fails.

Let us proceed with the proof of the converse. First, we assume that $F^{*}$ is bounded and $\mathcal{F}$ is lsc at $\pi$. By Theorem 4.2, parts $\mathrm{i}$ and ii, we conclude that $\vartheta$ is continuous at $\pi$ and then apply the converse statement in part i. If, alternatively, $F=F^{*}=\left\{x^{*}\right\}$, our first preliminary conclusion is that $\mathcal{F}$ is usc at $\pi$, and we shall check that the condition for the Hadamard well-posedness of $\pi$ is fulfilled in this case.

Let us consider an arbitrary sequence $\left\{\pi_{r}\right\} \subset \Pi_{b}$ converging to $\pi$. Lemma 4.1 applies again, yielding $m$ such that $\pi_{r} \in \Pi_{s}$ if $r \geq m$. Take $x^{r} \in F_{r}^{*}$ for $r \geq m$ and $x^{r} \in F_{r}$ if $r<m$. Then, $\left\{x^{r}\right\}$ is obviously an a.m.s. for $\pi$ associated with $\left\{\pi_{r}\right\}$.

The upper semicontinuity of $\mathcal{F}$ at $\pi$ implies that, given any open set $W$ containing $F=\left\{x^{*}\right\}$, there will exist an integer $r_{0}$ such that $F_{r} \subset W$ if $r \geq r_{0}$. In other words, $x^{r} \in W$ when $r \geq r_{0}$, and this means $\lim _{r} x^{r}=x^{*}$.

iii. Take $x^{*} \in F^{*}$ and $u \in O^{+}\left(F^{*}\right)$ with $\|u\|_{\infty}=1$. Then define $\mu_{r}=\frac{1}{u^{\prime} x^{*}+r}$, with $r$ sufficiently large to guarantee the positiveness of the denominator, and take $c^{r}:=c-\mu_{r} u$ and $y^{r}:=x^{*}+r u$. Obviously, $y^{r} \in F^{*}$ and $\left(c^{r}\right)^{\prime} y^{r}=v-1$.

Now let us define the systems

$$
\sigma_{r}:=\left\{\left(a_{t}+\frac{1}{k_{r}} c^{r}\right)^{\prime} x \geq b_{t}+\frac{v-1}{k_{r}}, t \in T\right\}, \quad r=1,2, \ldots,
$$


where the constants $k_{r}$ are chosen in such a way that

$$
\left\|\frac{c^{r}}{k_{r}}\right\|_{\infty}<\frac{1}{r} \quad \text { and } \quad\left|\frac{v-1}{k_{r}}\right|<\frac{1}{r}
$$

Finally, we shall introduce the associated problems $\pi_{r}:=\left(c^{r}, \sigma_{r}\right)$, which obviously verify $\lim _{r} \pi_{r}=\pi$ and $\pi_{r} \in \Pi_{c}$ (because $y^{r} \in F_{r}$ ).

If $\mathcal{F}$ is not lsc at $\pi$, condition iii in Theorem 3.1 will fail, and a sequence $\left\{\lambda^{p}\right\} \subset$ $\mathbb{R}_{+}^{(T)}$ exists verifying $\sum_{t \in T} \lambda_{t}^{p}=1, p=1,2, \ldots$, and (4.3). This implies, for each $r \in \mathbb{N}$,

$$
\lim _{p} \sum_{t \in T} \lambda_{t}^{p}\left(\begin{array}{c}
a_{t}+\frac{1}{k_{r}} c^{r} \\
b_{t}+\frac{v-1}{k_{r}}
\end{array}\right)=\frac{1}{k_{r}}\left(\begin{array}{c}
c^{r} \\
v-1
\end{array}\right)
$$

and the nonhomogeneous Farkas lemma allows us to conclude that $\left(c^{r}\right)^{\prime} x \geq v-1$ is a consequence of $\sigma_{r}$, which in fact entails $y^{r} \in F_{r}^{*}$ and $v_{r}=v-1$, contradicting part i.

Corollary 4.4. Let $\pi$ be a Hwp problem. If $x^{*}$ is the limit of a certain a.m.s, then $x^{*}$ will be optimal for $\pi$ (i.e., $x^{*} \in F^{*}$ ).

Proof. There must exist a sequence $\left\{\pi_{r}\right\} \subset \Pi_{b}$ converging to $\pi$, and an associated sequence $\left\{x^{r}\right\}, x^{r} \in \mathcal{F}\left(\pi_{r}\right)$ for every $r \in \mathbb{N}$, such that

$$
\lim _{r}\left\{\left(c^{r}\right)^{\prime} x^{r}-v_{r}\right\}=0 \text { and } \lim _{r} x^{r}=x^{*} .
$$

Statement $\mathrm{i}$ in Theorem 4.3 establishes the continuity of $\vartheta_{b}$ at $\pi$, entailing $\lim _{r} v_{r}=v$. Thus,

$$
0=\lim _{r}\left\{\left(c^{r}\right)^{\prime} x^{r}-v_{r}\right\}=c^{\prime} x^{*}-v
$$

at the same time that $x^{*}$ is feasible for $\pi$ since, for every $t \in T$,

$$
0 \leq \lim _{r}\left\{\left(a_{t}^{r}\right)^{\prime} x^{r}-b_{t}^{r}\right\}=a_{t}^{\prime} x^{*}-b_{t}
$$

(convergence in $(\Pi, \delta)$ yields $\lim _{r} c^{r}=c, \lim _{r} a_{t}^{r}=a_{t}$, and $\lim _{r} b_{t}^{r}=b_{t}$ for all $t \in T)$.

The only antecedents of the results presented in this section come from the field of continuous LSIP, and they can be traced out from [2] and the references therein. Most of the statements in [2, sects. 2 and 3] can be obtained as corollaries of Theorem 4.2 , emphasizing the fact that our results subsume all the previous contributions to the continuous problem. Additionally, the Lipschitzian condition given in $[2$, Thm. $3.5]$ is a trivial consequence of the inequality established in part iii of Theorem 4.2.

5. Optimal set mapping. The only theorem in this section concerns the stability behavior of $\mathcal{F}^{*}$.

ThEOREM 5.1. Given $\pi \in \Pi_{s}$, the following propositions hold:

i. $\mathcal{F}^{*}$ is closed at $\pi$ if and only if either $\mathcal{F}$ is lsc at $\pi$ or $F=F^{*}$.

ii. If $\mathcal{F}^{*}$ is usc at $\pi$, then $\mathcal{F}^{*}$ is closed at $\pi$. The converse statement holds if $F^{*}$ is bounded.

iii. $\mathcal{F}^{*}$ is lsc at $\pi$ if and only if $\mathcal{F}$ is lsc at $\pi$ and $F^{*}$ is a singleton.

Proof. i. Suppose that $\mathcal{F}^{*}$ is closed at $\pi$ and that, simultaneously, $F \neq F^{*}$ and $\mathcal{F}$ is not lsc at $\pi$. 
Let $y \in F \backslash F^{*}$. Then $c^{\prime} y=v+\alpha$ for a certain $\alpha>0$, and we shall consider a sequence of problems $\left\{\pi_{r}=\left(c, \sigma_{r}\right)\right\}$, where

$$
\sigma_{r}:=\left\{\left(a_{t}+r^{-1} c\right)^{\prime} x \geq b_{t}+r^{-1}(v+\alpha), t \in T\right\}, \quad r=1,2, \ldots
$$

It follows that $\lim _{r} \pi_{r}=\pi$, that $y \in F_{r}$ for all $r$, and that $c^{\prime} x \geq c^{\prime} y$ is a consequence of $\sigma_{r}$, again for every $r$ (we should apply the technique used in the proof of proposition ii in Theorem 4.3). This fact actually implies $y \in F_{r}^{*}, r=1,2, \ldots$, and the closedness of $\mathcal{F}^{*}$ at $\pi$ gives rise to the contradiction $y \in F^{*}$.

We continue with the proof of the converse statement. If $F=F^{*}$, we take sequences $\left\{\pi_{r}\right\}$ and $\left\{x^{r}\right\}$, converging to $\pi$ and $\bar{x}$, respectively, and also verifying $x^{r} \in F_{r}^{*}$. Since $F_{r}^{*} \subset F_{r}$ and $\mathcal{F}$ is always closed at $\pi$, one attains $\bar{x} \in F=F^{*}$.

Alternatively, if $\mathcal{F}$ is lsc at $\pi$ and we have $\lim _{r} \pi_{r}=\pi, \lim _{r} x^{r}=\bar{x}$, and $x^{r} \in F_{r}^{*}$, $r=1,2, \ldots$, we shall prove that $c^{\prime} \bar{x} \leq c^{\prime} x^{0}$ for any possible SS element of $\sigma, x^{0}$. First, we prove that $x^{0} \in F_{r}$ if $r \geq r_{0}$ for a certain $r_{0}$. Actually, if $\rho>0$ satisfies $a_{t}^{\prime} x^{0} \geq b_{t}+\rho$ for all $t \in T$, and $\delta\left(\pi_{0}, \pi\right)<\frac{\rho}{2} \min \left\{1, n^{-1 / 2}\left\|x^{0}\right\|^{-1}\right\}$ (writing $\left\|x^{0}\right\|^{-1}=+\infty$ in the case $x^{0}=0_{n}$ ), the Cauchy-Schwarz inequality leads us to

$$
\left(a_{t}^{0}\right)^{\prime} x^{0} \geq a_{t}^{\prime} x^{0}-\left\|x^{0}\right\|\left\|a_{t}^{0}-a_{t}\right\| \geq a_{t}^{\prime} x^{0}-\frac{\rho}{2} \geq b_{t}+\frac{\rho}{2} \geq b_{t}^{0} .
$$

Once we have established $x^{0} \in F_{r}$, if $r$ is sufficiently large, we write $\left(c^{r}\right)^{\prime} x^{r} \leq$ $\left(c^{r}\right)^{\prime} x^{0}$ and, taking limits for $r \rightarrow \infty, c^{\prime} \bar{x} \leq c^{\prime} x^{0}$ results.

Since $F$ is, in this case, the closure of the set of all the SS elements of $\sigma$ (condition vi in Theorem 3.1), one concludes that $c^{\prime} \bar{x} \leq c^{\prime} y$ for every feasible point $y \in F$, i.e., $\bar{x} \in F^{*}$.

ii. Since $(\Pi, \delta)$ behaves locally as the metric space $(\Pi, d)$ with $d\left(\pi_{1}, \pi\right)=\min$ $\left\{1, \delta\left(\pi_{1}, \pi\right)\right\}$, we can apply any property of set-valued mappings between metric spaces (see, for instance, [1]). In particular the upper semicontinuity of $\mathcal{F}^{*}$ at $\pi$ and the closedness of the set $F^{*}$ imply that $\mathcal{F}^{*}$ is a closed mapping at $\pi$.

In order to prove the converse statement, we assume that $F^{*}$ is bounded. If $F=F^{*}$, we have that $\mathcal{F}$ is usc at $\pi$, entailing the upper semicontinuity of $\mathcal{F}^{*}$ at the same problem $\pi$. When $\mathcal{F}$ is lsc at $\pi$, we use the following reasoning.

Let $W$ be an open set containing $F^{*}$, the last one being interpreted as the solution set of the system $\widetilde{\sigma}$ introduced in (4.1). The boundedness of $\widetilde{F} \equiv F^{*}$ implies the upper semicontinuity of $\widetilde{\mathcal{F}}$ at $\widetilde{\pi}:=(c, \widetilde{\sigma})$. In other words, $\eta_{1}>0$ exists such that $\widetilde{\delta}\left(\widetilde{\pi}_{1}, \widetilde{\pi}\right) \leq \eta_{1}$ guarantees $\widetilde{F}_{1} \subset W$. In particular, if we consider $\widetilde{\pi}_{1}:=\left(c, \widetilde{\sigma}_{1}\right)$, with

$$
\widetilde{\sigma}_{1}:=\left\{a_{t}^{\prime} x \geq b_{t}, \quad t \in T ; \quad c^{\prime} x \leq v+\eta_{1}\right\},
$$

we deduce the inclusion $\widetilde{F}_{1}=L\left(v+\eta_{1}\right) \subset W$.

Let $\bar{x}$ be an SS element of $\sigma$ (remember that $\mathcal{F}$ is lsc at $\pi$ ). If $c^{\prime} \bar{x}<v+\eta_{1}$, it is evident that $\bar{x}$ is an SS element of $\widetilde{\sigma}_{1}$ too. Otherwise, we pick $\widetilde{x} \in \widetilde{F}_{1}$ satisfying $c^{\prime} \widetilde{x}<v+\eta_{1}$. Then, if $\lambda$ is sufficiently small, $\lambda \bar{x}+(1-\lambda) \widetilde{x}$ will be an SS element of $\widetilde{\sigma}$. In any case, we conclude that $\widetilde{\mathcal{F}}$ is lsc at $\widetilde{\pi}_{1}$, implying the existence of $\eta_{2}>0$ such that $\widetilde{\delta}\left(\widetilde{\pi}_{2}, \widetilde{\pi}_{1}\right) \leq \eta_{2}$ leads us to $\widetilde{F}_{2} \neq \emptyset$.

Moreover, the boundedness of $\widetilde{F}_{1}=L\left(v+\eta_{1}\right)$ implies that $\widetilde{\mathcal{F}}$ is also usc at $\widetilde{\pi}_{1}$, and for a certain $\eta_{3}>0, \widetilde{\delta}\left(\widetilde{\pi}_{2}, \widetilde{\pi}_{1}\right) \leq \eta_{3}$ ensures $\widetilde{F}_{2} \subset W$.

Now, take a problem $\pi_{2}$ such that $\delta\left(\pi_{2}, \pi\right)<\eta:=\min \left\{\eta_{2}, \eta_{3}\right\}$, and let us associate with it the problem $\widetilde{\pi}_{2}:=\left(c^{2}, \widetilde{\sigma}_{2}\right)$ in $\widetilde{\Pi}$, with

$$
\widetilde{\sigma}_{2}:=\left\{\left(a_{t}^{2}\right)^{\prime} x \geq b_{t}^{2}, \quad t \in T ; \quad\left(c^{2}\right)^{\prime} x \leq v+\eta_{1}\right\} .
$$


TABLE 5.1

Stability and Hadamard well-posedness of the LSIP problem.

\begin{tabular}{|c|c|c|c|}
\hline \multicolumn{2}{|c|}{$F^{*}$ nonempty } & $\mathcal{F}$ lsc at $\pi$ & $\mathcal{F}$ non-lsc at $\pi$ \\
\hline \multirow{2}{*}{$\begin{array}{c}F^{*} \text { is a } \\
\text { singleton }\end{array}$} & $F=F^{*}$ & \multirow{2}{*}{$\begin{array}{c}\text { I,II,III,IV, } \\
\text { Hwp }\end{array}$} & $\overline{\mathrm{I}}, \mathrm{II}, \mathrm{III}, \overline{\mathrm{IV}}, \mathrm{Hwp}$ \\
\hline & $F \neq F^{*}$ & & $\overline{\mathrm{I}}, \overline{\mathrm{II}}, \mathrm{III}, \overline{\mathrm{IV}}, \overline{\mathrm{Hwp}}$ \\
\hline \multirow{2}{*}{$\begin{array}{l}F^{*} \text { is bounded, } \\
\text { not a singleton }\end{array}$} & $F=F^{*}$ & \multirow{2}{*}{$\begin{array}{c}\overline{\text { I}}, \mathrm{II}, \mathrm{III}, \mathrm{IV}, \\
\text { Hwp }\end{array}$} & $\overline{\mathrm{I}}, \mathrm{II}, \mathrm{III}, \overline{\mathrm{IV}}, \overline{\mathrm{Hwp}}$ \\
\hline & $F \neq F^{*}$ & & $\overline{\mathrm{I}}, \overline{\mathrm{II}}, \mathrm{III}, \overline{\mathrm{IV}}, \overline{\mathrm{Hwp}}$ \\
\hline \multirow{2}{*}{$\begin{array}{c}F^{*} \text { is } \\
\text { unbounded }\end{array}$} & $F=F^{*}$ & $\begin{array}{c}\text { Cell A: } \\
\overline{\mathrm{I}}, \overline{\mathrm{III}}_{c}, \mathrm{IV}\end{array}$ & $\overline{\mathrm{I}}, \frac{\text { Cell C: }}{\mathrm{III}}, \overline{\mathrm{IV}}, \overline{\mathrm{Hwp}}$ \\
\hline & $F \neq F^{*}$ & $\begin{array}{c}\text { Cell B: } \\
\overline{\mathrm{I}}, \overline{\mathrm{III}_{c}}, \mathrm{IV}\end{array}$ & $\overline{\mathrm{I}}, \overline{\mathrm{II}}, \overline{\mathrm{III}_{b}}, \overline{\mathrm{IV}}, \overline{\mathrm{Hwp}}$ \\
\hline
\end{tabular}

Obviously, $\widetilde{\delta}\left(\widetilde{\pi}_{2}, \widetilde{\pi}_{1}\right)<\eta$ and, consequently, $\emptyset \neq \widetilde{F}_{2}=L_{2}\left(v+\eta_{1}\right) \subset W$. Thus, $F_{2}^{*} \subset L_{2}\left(v+\eta_{1}\right) \subset W$ and the upper semicontinuity of $\mathcal{F}^{*}$ at $\pi$ follows.

iii. Let us suppose first that $F^{*}=\left\{x^{*}\right\}$ and that $\mathcal{F}$ is lsc at $\pi$. Then parts $\mathrm{i}$ and ii apply, and we conclude that $\mathcal{F}^{*}$ is usc at $\pi$.

Since $\mathcal{F}$ is lsc at $\pi$, there will exist $\eta_{1}>0$ such that $\delta\left(\pi_{1}, \pi\right)<\eta_{1}$ implies $\pi_{1} \in \Pi_{c}$. Lemma 4.1 allows us to write $\pi_{1} \in \Pi_{s}$ if $\eta_{1}$ is small enough.

Now take an open set $W$ containing $x^{*}$. The upper semicontinuity of $\mathcal{F}^{*}$ at $\pi$ gives rise to the existence of $\eta_{2}>0$ such that $\delta\left(\pi_{1}, \pi\right)<\eta_{2}$ implies $F_{1}^{*} \subset W$. If $\eta:=\min \left\{\eta_{1}, \eta_{2}\right\}$, one gets $\emptyset \neq F_{1}^{*} \subset W$, when $\delta\left(\pi_{1}, \pi\right)<\eta$, so that $F_{1}^{*} \cap W \neq \emptyset$ and $\mathcal{F}^{*}$ is certainly lsc at $\pi$.

Next we shall prove that the lower semicontinuity of $\mathcal{F}^{*}$ at $\pi$ implies that $\pi$ has a unique optimal solution. If this is not the case, we pick two different points in $F^{*}, x^{*}$, and $y^{*}$ and define $u:=y^{*}-x^{*}$. We shall introduce the sequence of problems $\pi_{r}:=$ $\left(c^{r}, \sigma\right), r=1,2, \ldots$, with $c^{r}:=c-\frac{1}{r} u$. Obviously, $\lim _{r} \pi_{r}=\pi$, and a contradiction will be attained.

Since $u^{\prime}\left(y^{*}-x^{*}\right)>0$, an open neighborhood of $x^{*}, W$, can be found such that $u^{\prime}\left(y^{*}-x\right)>0$ for every $x \in W$. Let us take an arbitrary $x \in W \cap F$, and notice that $\left(c^{r}\right)^{\prime}\left(y^{*}-x\right)=c^{\prime}\left(y^{*}-x\right)-\frac{1}{r} u^{\prime}\left(y^{*}-x\right)<0$. Hence $x \notin F_{r}^{*}$, and this contradicts the lower semicontinuity of $\mathcal{F}^{*}$ at $\pi$.

The last step in the proof will establish that the lower semicontinuity of $\mathcal{F}^{*}$ at $\pi$ implies that this property also holds for $\mathcal{F}$. Actually, we shall see that $\pi \in \operatorname{int}\left(\Pi_{c}\right)$. In fact, if $W$ is an open set such that $F^{*} \cap W$ is nonempty, there will exist $\eta>0$ such that $\delta\left(\pi_{1}, \pi\right)<\eta$ yields $F_{1}^{*} \cap W \neq \emptyset$, and $F_{1} \neq \emptyset$ in this neighborhood of $\pi$.

In [5, Thms. 3.3 and 4.2], the continuity properties of the optimal set mapping at a continuous solvable problem $\pi$ are analyzed. The optimal set mapping considered there is the restriction, $\mathcal{F}_{o s}^{*}$, of $\mathcal{F}^{*}$ to the subset of continuous solvable problems, $\Pi_{o s}$. So, the characterization of the lower semicontinuity of $\mathcal{F}_{o s}^{*}$ at $\pi \in \Pi_{o s}$ given in [5, Thm. 4.2] requires the existence of an extreme point of $F$ to guarantee the existence of solvable problems in a neighborhood of $\pi$.

Table 5.1 summarizes all the results presented in the previous sections. The following symbols are used: I $\Leftrightarrow \mathcal{F}^{*}$ is lsc at $\pi$; II $\Leftrightarrow \mathcal{F}^{*}$ is usc at $\pi$; III $\Leftrightarrow \vartheta$ is lsc at $\pi$; IV $\Leftrightarrow \vartheta$ is usc at $\pi ; \operatorname{III}_{b} \Leftrightarrow \vartheta_{b}$ is lsc at $\pi ; \operatorname{III}_{c} \Leftrightarrow \vartheta_{c}$ is lsc at $\pi\left(\left.\vartheta_{c} \equiv \vartheta\right|_{\Pi_{c}}\right) ; \overline{\mathrm{I}}$ means that I does not hold (etc.). 
6. Examples. By means of the following series of examples it is shown that in the cells of Table 5.1 associated with the cases " $F^{*}$ unbounded," every possible combination for the nonfixed properties can occur, showing that there is no additional underlying connection between them. All the examples are LSIP problems in $\mathbb{R}^{2}$, except Examples 6.3 and 6.4, which are posed in $\mathbb{R}^{3}$.

Cell A: $F^{*}$ unbounded, $F=F^{*}$, and $\mathcal{F}$ lsc at $\pi$

EXAMPLE 6.1. II and Hwp.

$$
\begin{aligned}
& \pi: \text { Inf } x_{1} \\
& \text { s.t. } t x_{1}+x_{2} \geq-1, t \in \mathbb{Z}, \\
& \quad s x_{2} \geq-1, \quad s \in \mathbb{N} .
\end{aligned}
$$

$F=F^{*}=\{0\} \times \mathbb{R}_{+}$and $v=0$. Moreover, $0_{2}$ is an SS element and $\mathcal{F}$ is lsc at $\pi$. If $\delta\left(\pi_{1}, \pi\right)<1$, we have $F_{1}^{*} \subset F_{1}=F=F^{*}$ and $\mathcal{F}^{*}$ is trivially usc at $\pi$. In order to prove that $\pi$ is Hwp, we have to establish the lower semicontinuity of $\vartheta_{b}$ at $\pi$, since this function is already usc at $\pi$ as a consequence of the lower semicontinuity of $\mathcal{F}$ at $\pi$. In fact, $\pi_{1} \in \Pi_{b}$ and $\delta\left(\pi_{1}, \pi\right)<1$ implies that $v_{1}$ is attained in the only extreme point of $F_{1}$, namely, $0_{2}$. In other words, $v_{1}=0$ and this entails the required continuity.

EXAMPLE 6.2. II and $\overline{\mathrm{Hwp}}$.

$$
\begin{aligned}
& \pi \text { : Inf } x_{1} \\
& \text { s.t. } t x_{1}+0 x_{2} \geq-1, t \in \mathbb{Z} .
\end{aligned}
$$

$F=F^{*}=\{0\} \times \mathbb{R}$ and $v=0$. Since $0_{2}$ is an SS element, $\mathcal{F}$ is lsc at $\pi$. If we define, for $r=1,2, \ldots$, the problem

$$
\begin{aligned}
\pi_{r}: \operatorname{Inf}\left(x_{1}+\frac{1}{r} x_{2}\right) \\
\quad \text { s.t. } t x_{1}+\frac{1}{r} x_{2} \geq-1, t \in \mathbb{Z},
\end{aligned}
$$

whose feasible set is $F_{r}=\{0\} \times\left[-r,+\infty\right.$ [, we observe that $\lim _{r} \pi_{r}=\pi$ and $v_{r}=-1$. Thus $\vartheta_{b}$ fails to be lsc at $\pi$.

EXAMPLE 6.3. $\overline{\mathrm{II}}$ and Hwp.

$$
\begin{aligned}
\pi: \operatorname{Inf} & x_{1} \\
\text { s.t. } & t x_{1}+x_{2}+x_{3} \geq-1, t \in \mathbb{Z}, \\
& x_{1}+s x_{2}+x_{3} \geq-1, \quad s \in \mathbb{N}, \\
& x_{1}+x_{2}+u x_{3} \geq-1, u \in \mathbb{N}, \\
& -x_{2}+x_{3} \geq-1 .
\end{aligned}
$$

$0_{3}$ is an SS element and, so, $\mathcal{F}$ is lsc at $\pi$. It can be seen that $x_{1} \geq 0,-x_{1} \geq 0$, $x_{2} \geq 0$, and $x_{3} \geq 0$ are consequent relations of the constraint system $\sigma$. To this end, we divide the first (second, third) block of constraints by $t(s, u)$ and take limits for $t \rightarrow \pm \infty(s \rightarrow+\infty, u \rightarrow+\infty)$. Conversely, the infinitely many constraints in the first three blocks are themselves consequences of $x_{1}=0, x_{2} \geq 0$, and $x_{3} \geq 0$. Consequently, $F=F^{*}=\left\{x \in \mathbb{R}^{3} \mid x_{1}=0, x_{2} \geq 0, x_{3} \geq 0, x_{3}-x_{2} \geq-1\right\}$.

If $\pi_{1}$ is a problem such that $\delta\left(\pi_{1}, \pi\right)<\varepsilon<1$, we can write it as follows:

$$
\begin{aligned}
& \pi_{1}: \operatorname{Inf}\left\{\left(1+\varepsilon_{1}\right) x_{1}+\varepsilon_{2} x_{2}+\varepsilon_{3} x_{3}\right\} \\
& \text { s.t. }\left(t+\varepsilon_{1}^{t}\right) x_{1}+\left(1+\varepsilon_{2}^{t}\right) x_{2}+\left(1+\varepsilon_{3}^{t}\right) x_{3} \geq-1+\varepsilon_{4}^{t}, \quad t \in \mathbb{Z}, \\
& \left(1+\varepsilon_{1}^{s}\right) x_{1}+\left(s+\varepsilon_{2}^{s}\right) x_{2}+\left(1+\varepsilon_{3}^{s}\right) x_{3} \geq-1+\varepsilon_{4}^{s}, \quad s \in \mathbb{N}, \\
& \left(1+\varepsilon_{1}^{u}\right) x_{1}+\left(1+\varepsilon_{2}^{u}\right) x_{2}+\left(u+\varepsilon_{3}^{u}\right) x_{3} \geq-1+\varepsilon_{4}^{u}, \quad u \in \mathbb{N}, \\
& \varepsilon_{1}^{w} x_{1}+\left(-1+\varepsilon_{2}^{w}\right) x_{2}+\left(1+\varepsilon_{3}^{w}\right) x_{3} \geq-1+\varepsilon_{4}^{w} \text {. }
\end{aligned}
$$


It is also obvious that $0_{3} \in F_{1}$ and that the first three blocks of constraints are still equivalent to the finite system $\left\{x_{1}=0, x_{2} \geq 0, x_{3} \geq 0\right\}$; i.e.,

$$
F_{1}=\left\{x \in \mathbb{R}^{3} \mid x_{1}=0, x_{2} \geq 0, x_{3} \geq 0 \text { and }\left(-1+\varepsilon_{2}^{w}\right) x_{2}+\left(1+\varepsilon_{3}^{w}\right) x_{3} \geq-1+\varepsilon_{4}^{w}\right\} .
$$

The first part of our argument consists in showing that $\mathcal{F}^{*}$ fails to be usc at $\pi$. Actually, if one introduces the approximating sequence (ap.s.) of problems $\pi_{r}$, $r=1,2, \ldots$, which differ from $\pi$ only in that the last constraint has been replaced by $-x_{2}+\left(1+\frac{1}{r}\right) x_{3} \geq-1$, respectively, it becomes evident that the open set $W=$ $\left\{x \in \mathbb{R}^{3} \mid-x_{2}+x_{3}>-2\right\}$ contains $F^{*}$, but $x^{r}=(0, r+2, r)^{\prime} \in F_{r} \backslash W=F_{r}^{*} \backslash W$. So, $F_{r}^{*} \nsubseteq W$ for every $r$ and $\mathcal{F}^{*}$ is not usc at $\pi$.

The second part is devoted to establishing the lower semicontinuity of $\vartheta_{b}$ at $\pi$. Since $F_{1}$ is a polyhedral set, if $\pi_{1} \in \Pi_{b}$, its optimal value will be attained in any of its two extreme points, namely,

$$
0_{3} \quad \text { and }\left(0, \frac{-1+\varepsilon_{4}^{w}}{-1+\varepsilon_{2}^{w}}, 0\right)^{\prime} ; \quad \text { i.e., } v_{1}=\min \left\{0, \frac{\varepsilon_{2}\left(\varepsilon_{4}^{w}-1\right)}{\varepsilon_{2}^{w}-1}\right\} \text {. }
$$

Accordingly, we shall write

$$
v_{1} \geq \frac{-\left|\varepsilon_{2}\right|\left(\varepsilon_{4}^{w}-1\right)}{\varepsilon_{2}^{w}-1}>\frac{-\varepsilon(1+\varepsilon)}{1-\varepsilon}
$$

and, since $\lim _{\varepsilon \rightarrow 0} \frac{\varepsilon(1+\varepsilon)}{1-\varepsilon}=0$, we conclude the intended property.

EXAMPLE 6.4. $\overline{\mathrm{II}}$ and $\overline{\mathrm{Hwp}}$.

$$
\begin{aligned}
& \pi: \operatorname{Inf} x_{1} \\
& \text { s.t. } t x_{1}+x_{2}+x_{3} \geq-1, \quad t \in \mathbb{Z} \text {, } \\
& x_{1}+s x_{2}+x_{3} \geq-1, \quad s \in \mathbb{N}, \\
& x_{1}+x_{2}+u x_{3} \geq-1, \quad u \in \mathbb{N}, \\
& -x_{2} \geq-1 \text {. }
\end{aligned}
$$

$0_{3}$ is an SS element and $F=F^{*}=\left\{x \in \mathbb{R}^{3} \mid x_{1}=0, x_{3} \geq 0\right.$ and $\left.x_{2} \in[0,1]\right\}$.

Now, let us introduce the ap.s. $\left\{\pi_{r}\right\}$ with $\pi_{r}$ differing from $\pi$ only in the last constraint, which is replaced by $-x_{2}+\frac{1}{r} x_{3} \geq-1$. Consider the open set $W=\{x \in$ $\left.\mathbb{R}^{3} \mid-x_{2}>-2\right\}$, the points $x^{r}=(0,2, r)^{\prime}, r=1,2, \ldots$, and observe that $W \supset F^{*}$ but $x^{r} \in F_{r} \backslash W=F_{r}^{*} \backslash W, r=1,2, \ldots$; i.e., $\mathcal{F}^{*}$ is not usc at $\pi$.

Next we prove that $\pi$ is not Hwp. Now we take into account the ap.s. $\left\{\tilde{\pi}_{r}\right\}$, such that $\widetilde{\pi}_{r}$ is obtained from changing the objective function of $\pi$ by $x_{1}-\frac{1}{r} x_{3}$ and substituting the last constraint of $\pi$ by $-x_{2}-\frac{1}{r} x_{3} \geq-1$. We get $\widetilde{F}_{r}=\operatorname{conv}\left\{0_{3}\right.$, $\left.(0,1,0)^{\prime},(0,0, r)^{\prime}\right\}$ and, consequently, $\widetilde{v}_{r}=-1, r=1,2, \ldots$; i.e., $\vartheta_{b}$ is not lsc at $\pi$.

Cell B: $F^{*}$ unbounded, $F \neq F^{*}$, and $\mathcal{F}$ lsc at $\pi$.

EXAMPLE 6.5. II and Hwp.

$$
\begin{array}{ll}
\pi: \text { Inf } & x_{1} \\
\text { s.t. } & t x_{1}+x_{2} \geq-1, \quad t \in \mathbb{N}, \\
& x_{1}+s x_{2} \geq-1, \quad s \in \mathbb{N} .
\end{array}
$$

$0_{2}$ is an SS element of $\pi$, entailing the lower semicontinuity of $\mathcal{F}$ at $\pi$. The constraints system is obviously equivalent to $x_{1} \geq 0$ and $x_{2} \geq 0$, so $F=\left\{x \in \mathbb{R}^{2} \mid x_{1} \geq 0\right.$, $\left.x_{2} \geq 0\right\}$ and $F^{*}=\{0\} \times \mathbb{R}_{+}$. In addition, if $\delta\left(\pi_{1}, \pi\right)<1$, we shall find $F_{1}=F$ and, since the objective function of $\pi_{1}$ is $\left(c^{1}\right)^{\prime} x=\left(1+\varepsilon_{1}\right) x_{1}+\varepsilon_{2} x_{2}$, one gets $\left(c^{1}\right)^{\prime}\left(\begin{array}{l}1 \\ 0\end{array}\right)=1+\varepsilon_{1}>0=\left(c^{1}\right)^{\prime} 0_{2}$. Hence, the point $(1,0)^{\prime}$ is not optimal for $\pi_{1}$, 
which implies that in the nontrivial case, namely, $\pi_{1} \in \Pi_{b}$, we have $\emptyset \neq F_{1}^{*} \subset F^{*}$; i.e., $\mathcal{F}^{*}$ is trivially usc at $\pi$. Simultaneously, $v_{1}=0=v$ and $\vartheta_{b}$ is obviously lsc at $\pi$.

EXAMPLE 6.6. II and $\overline{\mathrm{Hwp}}$.

$$
\begin{aligned}
\pi: \text { Inf } & x_{1} \\
\text { s.t. } & t x_{1}+x_{2} \geq-1, \quad t \in \mathbb{N}, \\
& x_{1}+s x_{2} \geq-1, \quad s \in \mathbb{N}, \\
& -x_{1} \geq-1 .
\end{aligned}
$$

$\mathcal{F}$ is lsc at $\pi$ because $0_{2}$ is, once more, an SS element. It can be easily verified that $F=[0,1] \times \mathbb{R}_{+}$and $F^{*}=\{0\} \times \mathbb{R}_{+}$. If $\delta\left(\pi_{1}, \pi\right)<1$ we have $c^{1}=\left(1+\varepsilon_{1}, \varepsilon_{2}\right)^{\prime}$ and $F_{1}=\left\{x \in \mathbb{R}^{2} \mid x_{1} \geq 0, x_{2} \geq 0\right.$ and $\left.\left(-1+\varepsilon_{1}^{\omega}\right) x_{1}+\varepsilon_{2}^{\omega} x_{2} \geq-1+\varepsilon_{3}^{\omega}\right\}$. We shall distinguish two cases:

i. $\varepsilon_{2}^{\omega}<0$. Then

$$
F_{1}=\operatorname{conv}\left\{0_{2},\left(\frac{1-\varepsilon_{3}^{\omega}}{1-\varepsilon_{1}^{\omega}}, 0\right)^{\prime},\left(0, \frac{1-\varepsilon_{3}^{\omega}}{-\varepsilon_{2}^{\omega}}\right)^{\prime}\right\} .
$$

ii. $\varepsilon_{2}^{\omega} \geq 0$. Now $F_{1}$ is unbounded, with two extreme points,

$$
0_{2} \quad \text { and } \quad\left(\frac{1-\varepsilon_{3}^{\omega}}{1-\varepsilon_{1}^{\omega}}, 0\right)^{\prime} \text {. }
$$

In any case, if $\pi_{1} \in \Pi_{s}$ (or, equivalently, $\pi_{1} \in \Pi_{b}$ since $\pi_{1}$ is equivalent to an ordinary linear programming problem), the optimal value is attained at some extreme point. Notice that

$$
\left(\frac{1-\varepsilon_{3}^{\omega}}{1-\varepsilon_{1}^{\omega}}, 0\right)^{\prime}
$$

will never be optimal, because

$$
\left(c^{1}\right)^{\prime} 0_{2}=0<\left(1+\varepsilon_{1}\right) \frac{1-\varepsilon_{3}^{\omega}}{1-\varepsilon_{1}^{\omega}}
$$

(remember that all the epsilons have absolute values smaller than 1). Consequently, $v_{1}$ will be attained at points with the first coordinate equal to zero; i.e., $F_{1}^{*} \subset F^{*}$ and $\mathcal{F}^{*}$ turns out to be usc at $\pi$.

Let us proceed, providing an ap.s. of problems for $\pi,\left\{\pi_{r}\right\}$, such that $v_{r}=-1$, $r=1,2, \ldots$, and, accordingly, $\vartheta_{b}$ will not be lsc at $\pi$. The problem $\pi_{r}$ is derived from $\pi$, replacing the objective function by $\left(c^{r}\right)^{\prime} x=x_{1}-\frac{1}{r} x_{2}$ and the last constraint by $-x_{1}-\frac{1}{r} x_{2} \geq-1$. Since $F_{r}=\operatorname{conv}\left\{0_{2},(1,0)^{\prime},(0, r)^{\prime}\right\}, v_{r}=\left(c^{r}\right)^{\prime}(0, r)^{\prime}=-1$ results.

EXAMPLE 6.7. $\overline{\mathrm{II}}$ and Hwp.

$$
\begin{array}{ll}
\pi: & \operatorname{Inf} x_{1} \\
\text { s.t. } & x_{1}+0 x_{2} \geq 0 .
\end{array}
$$

$x^{0}=(1,0)^{\prime}$ is an SS element, $F=\mathbb{R}_{+} \times \mathbb{R}$, and $F^{*}=\{0\} \times \mathbb{R}$.

Consider the approximating problem $\pi_{r}:=\operatorname{Inf}\left\{x_{1}+\frac{1}{r} x_{2} \mid x_{1}+\frac{1}{r} x_{2} \geq 0\right\}$, for which $\delta\left(\pi_{r}, \pi\right)=\frac{1}{r}$. Taking the open set $W:=\left\{x \in \mathbb{R}^{2} \mid x_{1}>-1\right\}$ and the points $x^{r}:=(-1, r)^{\prime}$, one has $W \supset F^{*}$ but $x^{r} \in F_{r}^{*} \backslash W$, and the upper semicontinuity of $\mathcal{F}^{*}$ does not hold at $\pi$.

In the following step, the Hadamard well-posedness of $\pi$ is shown. If $\pi_{1}$ is any problem obtained by perturbation of $\pi$, and $\delta\left(\pi_{1}, \pi\right)<\varepsilon<1$, we can write

$$
\pi_{1}:=\operatorname{Inf}\left\{\left(1+\varepsilon_{1}\right) x_{1}+\varepsilon_{2} x_{2} \mid\left(1+\varepsilon_{1}^{1}\right) x_{1}+\varepsilon_{2}^{1} x_{2} \geq \varepsilon_{3}^{1}\right\},
$$


with all the parameters having values in $]-1,1\left[\right.$. Then, $\pi_{1} \in \Pi_{b}$ if and only if

$$
\frac{1+\varepsilon_{1}}{1+\varepsilon_{1}^{1}}=\frac{\varepsilon_{2}}{\varepsilon_{2}^{1}}
$$

in which case

$$
v_{1}=\varepsilon_{3}^{1} \frac{1+\varepsilon_{1}}{1+\varepsilon_{1}^{1}} \quad \text { and } \quad v_{1} \geq v-\frac{\varepsilon(1+\varepsilon)}{1-\varepsilon} .
$$

Since $\lim _{\varepsilon \rightarrow 0} \frac{\varepsilon(1+\varepsilon)}{1-\varepsilon}=0, \vartheta_{b}$ comes to be lsc at $\pi$.

EXAMPLE 6.8. $\overline{\mathrm{II}}$ and $\overline{\mathrm{Hwp}}$.

$$
\begin{array}{cc} 
& \pi: \operatorname{Inf} x_{1} \\
\text { s.t. } & x_{1}+0 x_{2} \geq 0, \\
- & x_{1}+0 x_{2} \geq-1 .
\end{array}
$$

$x^{0}=\left(\frac{1}{2}, 0\right)^{\prime}$ is an SS element, $F=[0,1] \times \mathbb{R}$, and $F^{*}=\{0\} \times \mathbb{R}$. On this occasion, $\pi_{r}:=\operatorname{Inf}\left\{x_{1}+\frac{1}{r} x_{2} \mid x_{1}+\frac{1}{r} x_{2} \geq 0,-x_{1} \geq-1\right\}$, and the argument uses exactly the same terms as in the previous example to conclude that $\mathcal{F}^{*}$ is not usc at $\pi$.

In order to check that $\pi$ is not Hwp, take $\widetilde{\pi}_{r}:=\operatorname{Inf}\left\{x_{1}-\frac{1}{r} x_{2} \mid x_{1}-\frac{1}{2 r} x_{2} \geq\right.$ $\left.0,-x_{1} \geq-1\right\}$. Note that $\delta\left(\widetilde{\pi}_{r}, \pi\right)=\frac{1}{r}$ and $\left\{\widetilde{\pi}_{r}\right\}$ is an ap.s. for $\pi$. Moreover, $\widetilde{F}_{r}^{*}=$ $\left\{(1,2 r)^{\prime}\right\}$ and $\widetilde{v}_{r}=-1$, precluding the lower semicontinuity of $\vartheta_{b}$ at $\pi$.

Cell C: $F^{*}$ unbounded, $F=F^{*}$, and $\mathcal{F}$ non-lsc at $\pi$.

EXAMPLE 6.9. II.

$$
\begin{array}{ll}
\pi: & \operatorname{Inf} x_{1} \\
\text { s.t. } & t x_{1}+0 x_{2} \geq-1, \quad t \in \mathbb{Z}, \\
& x_{1}+0 x_{2} \geq 0, \\
& -x_{1}+0 x_{2} \geq 0 .
\end{array}
$$

$F=F^{*}=\{0\} \times \mathbb{R}$. There is no SS element and, so, $\mathcal{F}$ is not lsc at $\pi$. If $\delta\left(\pi_{1}, \pi\right)$ is finite, $F_{1}^{*} \subset F_{1} \subset F=F^{*}$, implying II.

EXAMPLE 6.10. II.

$$
\begin{array}{ll}
\pi: & \operatorname{Inf} x_{1} \\
\text { s.t. } & x_{1}+0 x_{2} \geq 0, \\
& -x_{1}+0 x_{2} \geq 0 .
\end{array}
$$

$F=F^{*}=\{0\} \times \mathbb{R}$, and we have no SS element. Defining $\pi_{r}:=\operatorname{Inf}\left\{x_{1}+\frac{1}{r} x_{2} \mid\right.$ $\left.x_{1}+\frac{1}{r} x_{2} \geq 0,-x_{1}-\frac{1}{r} x_{2} \geq 0\right\}$, one has $\delta\left(\pi_{r}, \pi\right)=\frac{1}{r}, x^{r}:=(-1, r)^{\prime} \in F_{r}^{*}$, but $x^{r} \notin W:=\left\{x \in \mathbb{R}^{2} \mid x_{1}>-1\right\} \supset F^{*}$. This yields II.

Acknowledgments. The authors wish to thank M. A. Goberna for his many useful suggestions and the anonymous referees for their valuable critical comments.

\section{REFERENCES}

[1] B. Brosowski, Parametric Semi-Infinite Optimization, Verlag Peter Lang, Frankfurt-AmMain, Germany, 1982.

[2] B. Brosowski, Parametric semi-infinite linear programming I. Continuity of the feasible set and of the optimal value, Math. Programming Stud., 21 (1984), pp. 18-42.

[3] M. J. Cánovas, M. A. López, J. Parra, and M. I. Todorov, Solving Strategies and WellPosedness in Linear Semi-Infinite Programming, Dept. of Statistics and Operations Research Working Paper Series, University of Alicante, Alicante, Spain, 1998.

[4] A. L. Dontchev and T. Zolezzi, Well-Posed Optimization Problems, Springer-Verlag, Berlin, 1993. 
[5] T. Fischer, Contributions to semi-infinite linear optimization, in Approximation and Optimization in Mathematical Physics, B. Brosowski and E. Martensen, eds., Verlag Peter Lang, Frankfurt-Am-Main, Germany, 1983, pp. 175-199.

[6] M. A. Goberna And M. A. López, Topological stability of linear semi-infinite inequality systems, J. Optim. Theory Appl., 89 (1996), pp. 227-236.

[7] M. A. Goberna, M. A. López, And M. I. Todorov, Stability theory for linear inequality systems, SIAM J. Matrix Anal. Appl., 17 (1996), pp. 730-743.

[8] M. A. Goberna, M. A. López, And M. I. Todorov, Stability theory for linear inequality systems II: Upper semicontinuity of the solution set mapping, SIAM J. Optim., 7 (1997), pp. $1138-1151$.

[9] R. Henrion and D. Klatte, Metric regularity of the feasible set mapping in semi-infinite optimization, Appl. Math. Optim., 30 (1994), pp. 103-109.

[10] M. A. JimÉnez And J. J. RÜCKMAnN, On equivalent stability properties in semi-infinite optimization, Z. Oper. Res., 41 (1995), pp. 175-190.

[11] H. Th. Jongen, F. Twilt, and G. W. Weber, Semi-infinite optimization: Structure and stability of the feasible set, J. Optim. Theory Appl., 72 (1992), pp. 529-552.

[12] H. Th. Jongen, J.-J. RÜCKMAnn, And G.-W. Weber, One-parametric semi-infinite optimization: On the stability of the feasible set, SIAM J. Optim., 4 (1994), pp. 637-48.

[13] D. KLATte, Stable local minimizers in semi-infinite optimization: Regularity and second-order conditions, J. Comput. Appl. Math., 56 (1994), pp. 137-57.

[14] S. M. Robinson, Stability theory for systems of inequalities. Part I: Linear systems, SIAM J. Numer. Anal., 12 (1975), pp. 754-769.

[15] R. T. Rockafellar and R. J.-B. Wets, Variational Analysis, Springer-Verlag, Berlín, 1998.

[16] J. J. RüCKmann, Topological stability of feasible sets in semi-infinite optimization: A tutorial, Institut für Geometrie und Praktische Mathematik, RWTH Aachen, Germany, 1995.

[17] M. I. Todorov, Generic existence and uniqueness of the solution set to linear semi-infinite optimization problems, Numer. Funct. Anal. Optim., 8 (1985/1986), pp. 27-39.

[18] H. TuY, Stability property of a system of inequalities, Math. Oper. Statist. Ser. Opt., 8 (1977), pp. 27-39.

[19] Y. J. ZHU, Generalizations of some fundamental theorems on linear inequalities, Acta Math. Sinica, 16 (1966), pp. 25-40. 